\title{
Progressive familial myoclonus epilepsy
}

\author{
H. RUSTAM, T. HAMDI, AND S. WITRI \\ From the Department of Neurology and Department of Neurosurgery, \\ Medical City Teaching Hospital, College of Medicine, University of Baghdad, Iraq
}

SYNOPSIS Seven cases of progressive familial myoclonus epilepsy occurring in three families are presented. The patients were in different stages of the illness. The EEG was abnormal in all. It is suggested that these cases belong clinically to the Lafora bodies group. Nystagmus and optic atrophy, seen in one patient, have not been described previously. Myoclonic jerks did not respond to treatment with diazepam and ethosuximide.

Progressive familial myoclonus epilepsy, a rare disease, was first described in five siblings by Unverricht (1891). Grand mal fits, myoclonic jerks, and progressive mental and physical deterioration are the main clinical features. Lundborg (1913) reported similar cases and divided the disease process into three stages: (1) epileptic, (2) myoclonic, (3) terminal.

We report seven cases occurring in three families.

\section{FAMILY A, CASE 1}

I.R., an 18 year old girl, had a normal birth and development. She started school at the age of six years. Myoclonic jerks appeared at 10 years of age and were localized to the upper limbs. They became more frequent and generalized and caused her to fall. Within a few months, attacks of loss of consciousness developed and she had to leave school. The myoclonic jerks were present during sleep and increased under stress. They were precipitated by sudden loud sound and excitement. On examination (10 March 1973) ataxia was her only abnormality. Investigations including skull radiographs, CSF analysis, complete blood picture, liver function tests, and serum electrolytes were normal. The EEG showed paroxysmal spikes, multispikes, and spike/ sharp wave complexes which were localized and diffuse (Fig. 1).

\section{CASE A2}

B.R., aged 25 years, was the sister of case A1. Birth and development were normal. Her illness started as

(Accepted 29 April 1975.) falling attacks at 15 years of age which were followed by grand mal fits. After several months myoclonic jerks appeared and became progressively more frequent and generalized. She was ataxic in her gait, dysarthric, and dropped objects. During the next five years she deteriorated and became bedridden, incontinent of urine and faeces, speechless, and inaccessible. Her vision and hearing were apparently preserved. Neurological assessment was difficult but the fundi were normal. She died in September 1974.

\section{CASE A3}

T.R., brother of cases A1 and A2, died 10 years ago when aged 25 years. Attacks of falling started at 10 years of age and were followed by grand mal fits and myoclonic jerks. He deteriorated progressively and was bedridden until his death.

FAMILY B, CASE 1

J.M., a 15 year old boy, of normal birth and development, had started school at 6 years of age. When he was 10 years old myoclonic jerks appeared which were irregular, asymmetrical, and localized to the extremities. The jerks became more frequent and began to spread, rapidly involving the whole body and ending in generalized convulsions and loss of consciousness. The jerks occurred during sleep and wakefulness. During the last two years he was unsteady in his gait and dysarthric. Myoclonic jerks continued to be irregular but became symmetrical and involved the head and the trunk. They were precipitated by sudden loud sounds. For the last six months he was bedridden. On examination (1 July 1973) he lay in bed in generalized flexion and resisted 


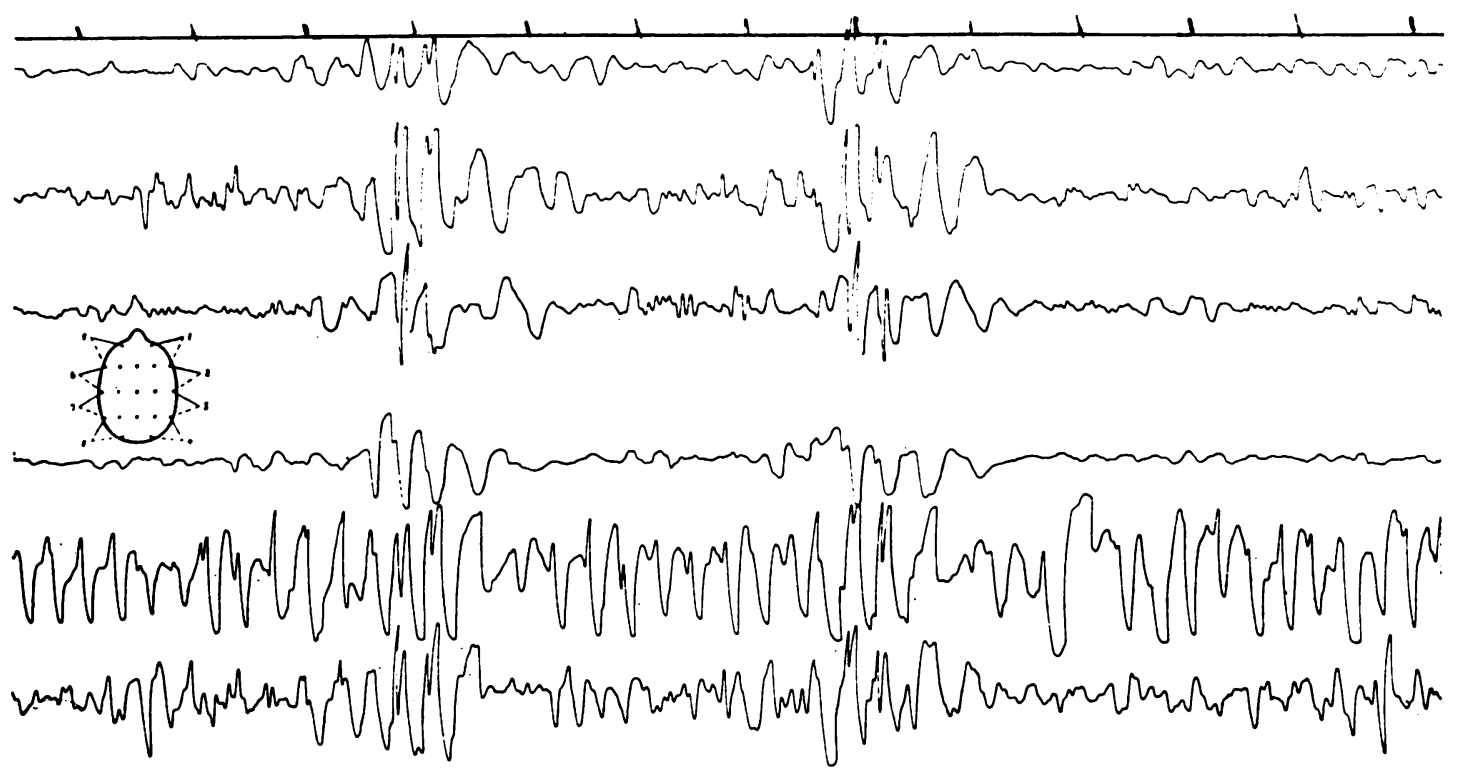

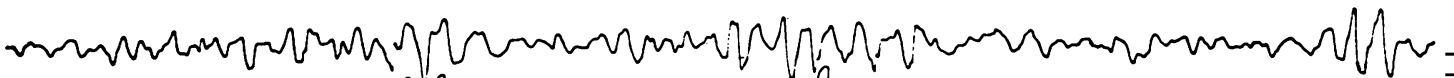

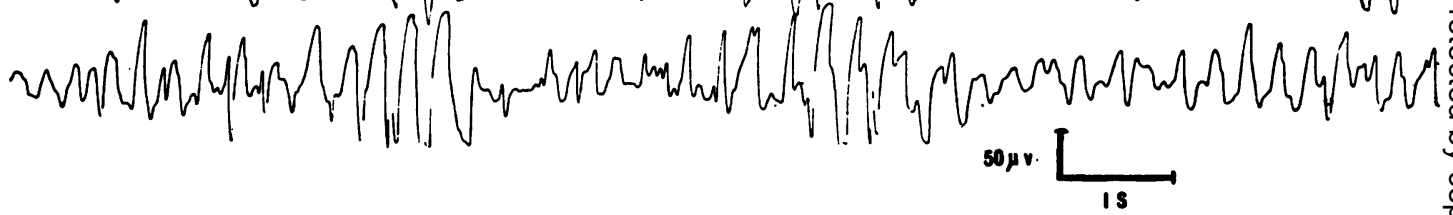

FIG. 1 Case 1, family A (I.R.). EEG showing localized and diffuse spikes, multispikes, and spike and sharp waves complexes especially on the left side.

any interference. Attempts to examine him induced generalized myoclonic jerks with twitching of the facial muscles. Neurological examination was impossible. He died in July 1974 and two months before his death he lost his vision. All investigations were normal. The EEG showed bilateral spikes and sharp waves on a low amplitude background activity.

\section{CASE B2}

H.M., a girl aged 10 years, sister of case B1, had normal birth and development and her illness started when she was 9 years old as unsteadiness with slurred speech and frequent dropping of objects. Attacks of loss of consciousness and generalized convulsions developed later. During the last five months myoclonic jerks of the upper and lower limbs appeared; at times these were so severe that the patient was thrown to the floor. The jerks were irregular and persisted during sleep. On examination
(3 July 1973) she was alert and cooperative but afraid of walking unaided because of the sudden falling attacks. Her gait was broad-based, speech was slurred, and she had intention tremor. There was bilateral horizontal jerking nystagmus, especially on looking to the left. Muscle tone and tendon reflexes were normal. When re-examined in December 1974 her visual acuity was reduced to finger counting and she had bilateral optic atrophy. Speech was very slurred, tendon jerks were brisk, but the plantar reflexes were flexor. Myoclonic jerks were induced by passive and active movement, by touch, and even on attempting to speak. Investigations were normal, except for the EEG which showed bilaterally synchronous spike activity.

\section{FAMILY C, CASE 1}

S.M. was a girl aged 16 years with normal birth and development. At 13 years of age myoclonic jerks of 

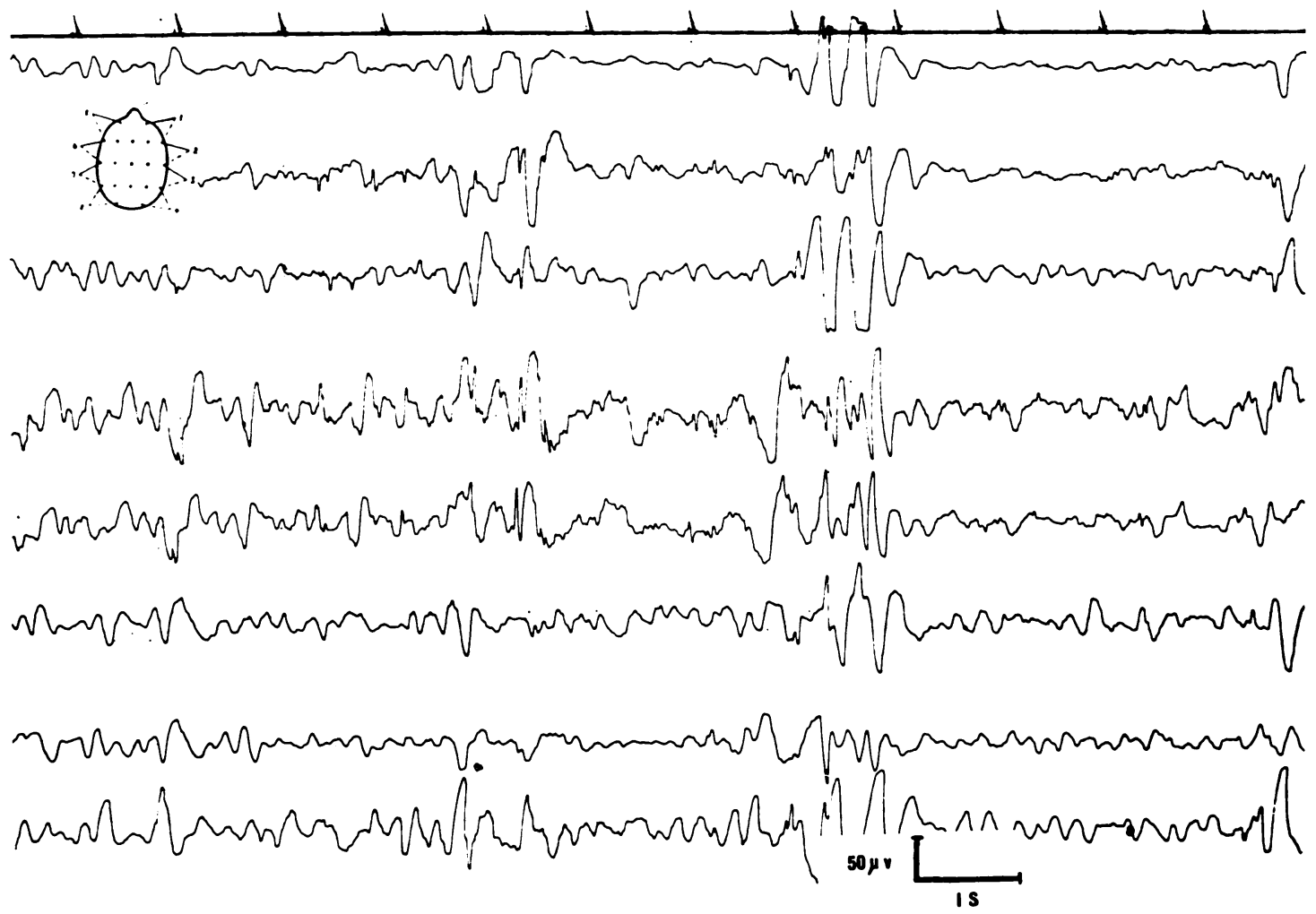

FIG. 2 Case 2, family C (A.M.). EEG showing localized and diffuse spikes, sharp waves, and spike and sharp waves complexes.

upper and lower limbs started. The jerks were asymmetrical and initiated by voluntary movements and by walking. Gradually myoclonic jerks became more frequent, more severe and generalized. They disappeared during sleep. Later, attacks of grand mal fits appeared. On examination she was alert, cooperative, and mentally clear. Her speech was normal but her gait was broad-based and moderately ataxic. Myoclonic jerks interfered with examination. There was no evidence of pyramidal tract lesion and vision was normal. Investigations were normal and the EEG resembled the other cases.

\section{CASE C2}

A.M., sister of case $\mathrm{Cl}$, was a 17 year old girl who had normal birth and development. Her illness started at the age of 12 years as myoclonic jerks affecting the upper and lower limbs. The jerks increased on attempting to hold objects. They gradually increased in severity and interfered with walking and occasionally threw the patient to the ground. On examination (9 February 1973) she was fully alert and mentally lucid. Speech and cranial nerves were intact. Tendon jerks were normal but Babinski's response was present on the left side. Coordination was impaired in the upper and lower limbs and her gait was ataxic. The myoclonic jerks made examination difficult but they were absent during sleep. Investigations were normal except for a characteristic EEG (Fig. 2).

\section{DISCUSSION}

Progressive familial myoclonus epilepsy as described by Unverricht (1891) is now recognized as a distinct clinical entity. It is characterized by grand mal epilepsy, myoclonus, and progressive deterioration. On histopathological grounds, Harriman and Millar (1955) were able to classify the cases collected from the literature into three groups:

1. Lafora bodies group: there was diffuse 
distribution of intracellular Lafora bodies in the central nervous system.

2. Cases with lipid inclusion bodies: the intracellular inclusion bodies consisted of lipoid material.

3. Degenerative group: these cases showed degeneration of the neurones and tracts of the central nervous system.

In this paper, seven cases of progressive familial myoclonic epilepsy occurring in three families are described. In family A three siblings out of five were affected, two of whom were seen by the authors and the other died 10 years ago. The onset was at between 10 to 15 years of age and started as falling attacks followed by grand mal fits and then myoclonic jerks. Cases 2 and 3 died in a demented state. Evidence of cerebellar impairment was present in all three. The parents were not related and were free from nervous system disorder. In family $B$ the age of onset was between 9 and 10 years and the illness started as myoclonic jerks in one patient and as a cerebellar disorder in the other. In both of them the myoclonic jerks increased in amplitude and ended in generalized convulsion and loss of consciousness. Case B1 died last year and case $\mathrm{B} 2$ is deteriorating. The parents were first cousins and in good health. In family $\mathrm{C}$, the age of onset was between 12 to 13 years and the illness started as myoclonic jerks followed by ataxia and dysarthria. Grand mal fits were present in one patient and pyramidal system defect in the other. The parents are second cousins and in good health.

Our cases fulfil most of the criteria suggested by Schwartz and Yanoff (1965) and Janeway et al. (1967). The onset was in adolescence, consanguinity between parents was common, and clinically there were myoclonus, grand mal fits, and cerebellar defect. Some of the patients reached the terminal stages and died within six to 10 years from the onset, others are progressively deteriorating, and the rest are in the early phase of the syndrome. Nystagmus and optic atrophy, seen in one patient, have not been described in other studies. All cases were in one generation and the parents were free from nervous system disease.

In all patients the EEG was abnormal, showing $\stackrel{0}{\stackrel{0}{2}}$ localized or diffuse spikes, multispikes, or spike and sharp wave complexes. Unfortunately, no histopathological findings are available but on clinical grounds we suggest that these cases belong to the Lafora bodies group of progressive familial myoclonus epilepsy. Watson and DennyBrown (1953), however, found neuronal lipidosis and Matthews et al. (1969) did not find Lafora bodies in their similar cases.

The grand mal fits were fairly well controlled by anticonvulsants but the myoclonus did not respond to treatment or improved slightly with diazepam and ethosuximide. The myoclonic jerks became worse during the terminal stages of the illness. Lope et al. (1974) reported promising results with clonazepam for the control of myoclonus.

\section{REFERENCES}

Harriman, D. G. F., and Millar, J. H. D. (1955). Progressive familial myoclonus epilepsy in three families. Its clinica features and pathological basis. Brain, 78, 325-348.

Janeway, R., Raven, J. R., Pearce, L. A., Odar, D. L., and Suzuki, K. (1967). Progressive myoclonus epilepsy with్ Lafora inclusion bodies. Archives of Neurology (Chic.), 16.2. 565-582.

Lope, E. S., Junquera, S. R., and Berenguel, A. B. (1974). Progressive myoclonic epilepsy with Lafora bodies. Clinicopathological study. Acta Neurologica Scandinavica, 50, 537-552.

Lundborg, H. (1913). Medizinisch-Biologische Familienforschungen, Innerhalb eines 2232-Kopfigen Baweryeschleschtes in Schweden. Jena.

Matthews, W. B., Howel, D. A., and Stevens, D. L. (1969). Progressive myoclonus epilepsy without Lafora bodies. Journal of Neurology, Neurosurgery, and Psychiatry, 32. 116-122.

Schwartz, G. A., and Yanoff, M. (1965). Lafora disease. Distinct clinico-pathologic form of Unverricht's syndrome. Archives of Neurology (Chic.), 12, 172-188.

Unverricht, H. (1891). Die Myoklonie. Deutche: Leipzig.

Watson, C. D., and Denny-Brown, D. (1953). Myoclonus epilepsy as a symptom of diffuse neuronal disease. Archives of Neurology and Psychiatry (Chic.), 70, 151-168. 